\title{
Enduring neurobehavioral effects induced by microbiota depletion during the adolescent period
}

\author{
Gilliard Lach (1) ${ }^{1,6}$, Christine Fülling ${ }^{1}$, Thomaz F. S. Bastiaanssen (1) ${ }^{1,2}$, Fiona Fouhy ${ }^{1,3}$, Aoife N. O' Donovan 1,3,4, \\ Ana Paula Ventura-Silva', Catherine Stanton ${ }^{1,3}$, Timothy G. Dinan ${ }^{1,5}$ and John F. Cryan (1) ${ }^{1,2}$
}

\begin{abstract}
The gut microbiota is an essential regulator of many aspects of host physiology. Disruption of gut microbial communities affects gut-brain communication which ultimately can manifest as changes in brain function and behaviour. Transient changes in gut microbial composition can be induced by various intrinsic and extrinsic factors, however, it is possible that enduring shifts in the microbiota composition can be achieved by perturbation at a timepoint when the gut microbiota has not fully matured or is generally unstable, such as during early life or ageing. In this study, we investigated the effects of 3-week microbiota depletion with antibiotic treatment during the adolescent period and in adulthood. Following a washout period to restore the gut microbiota, behavioural and molecular hallmarks of gut-brain communication were investigated. Our data revealed that transient microbiota depletion had long-lasting effects on microbiota composition and increased anxiety-like behaviour in mice exposed to antibiotic treatment during adolescence but not in adulthood. Similarly, gene expression in the amygdala was more severely affected in mice treated during adolescence. Taken together these data highlight the vulnerability of the gut microbiota during the critical adolescent period and the long-lasting impact manipulations of the microbiota can have on gene expression and behaviour in adulthood.
\end{abstract}

\section{Introduction}

The adolescent period is a key developmental period which marks the transition from childhood to adulthood. It is during this last developmental stage before adulthood that the brain is highly responsive to certain environmental cues that will shape neuronal architecture and promote maturation of social behaviours, emotional and cognitive capabilities and is hence a vulnerable period for the onset of psychiatric diseases ${ }^{1}$. The gut microbiota composition of an adolescent is usually simpler and more unstable when compared with that of an adult, which is highly diverse and stable ${ }^{2,3}$. These differences are probably due to relative

\footnotetext{
Correspondence: John F. Cryan (j.cryan@ucc.ie)

${ }^{1}$ APC Microbiome Ireland, University College Cork, Cork, Ireland

${ }^{2}$ Department of Anatomy and Neuroscience, University College Cork, Cork, Ireland

Full list of author information is available at the end of the article

These authors contributed equally: Gilliard Lach, Christine Fülling
}

immaturity of the gut microbiota during the adolescent period, which makes it more vulnerable to environmental stressors such as infection, use of antibiotic and poor diet. In addition to this, gonadal hormones are peaking during the puberty and it has been shown to have a long-term on the microbiota diversity ${ }^{1,4}$. Overt changes in the gut microbiota composition might therefore contribute to the onset of such disease and could be targeted by the use of biotherapeutics, antibiotics or different types of $\operatorname{diet}^{2,5,6}$. Nonetheless, the consequences of gut microbiota manipulation during adolescence is yet to be fully understood.

The gastrointestinal tract is colonised by trillions of bacteria that are tightly associated with host physiology. When the equilibrium of the microbial milieu in the gut is shifted it can have long-lasting effects on whole-body health including the brain and behaviour ${ }^{7-11}$. In mammals, the initial microbiota is obtained during the birthing process and develops alongside its host from a rather 
instable to a highly stable and diverse community in adulthood $^{2,12}$. Microbiota composition during the developmental period is shaped by a combination of genetic and environmental factors to be highly adapted to the host and the host's environment ${ }^{12}$. However, maladaptation of the gut microbiota could affect key innate and adaptative immune signalling within the intestine and at sites anatomically remote such as the brain resulting in altered host response to infection and vaccination as well as increase the susceptibility to brain disorders ${ }^{2,13}$. For example, altered gut microbiota composition can immediately affect brain function by impacting the turnover and release of neurotransmitters, hormones as well as growth factors and consequently affect behavioural parameters and thereby increase the susceptibility to develop neuropsychiatric disorders ${ }^{5,14-17}$.

One way to study the perturbation of the gut microbiota during critical periods is by using antibioticinduced depletion. Antibiotics are one of the most important factors influencing the gut microbiota composition and structure. Studies in both female and male mice and rats ${ }^{16,18-20}$ and humans ${ }^{21,22}$ have shown that antibiotic administration can induce changes in physiology, brain and behaviour. Antibiotic depletion of the microbiota for a defined time period represents an advantage in comparison to classic approach such as germ-free (GF) animals as the effects of microbiota depletion during the early developmental period can be avoided $^{23,24}$. It has been shown, that the use of antibiotics to chronically deplete the intestinal microbiota during adulthood has been associated with hormonal changes and alterations in gene expression, decreased adult hippocampal neurogenesis, and changes anxietyrelated responses, exploratory behaviour, and cognitive abilities $^{20,25,26}$. Moreover, early life exposure to antibiotics induces long-lasting increases in visceral pain responses $^{27}$ as well as altered metabolic programming ${ }^{18}$. We have previously shown that microbiota depletion with antibiotics commencing in adolescence all the way through adulthood resulted in deficits in anxiety and cognitive behaviour ${ }^{25}$. It is not clear whether such changes are a result of microbiota changes specifically in adolescence, in adulthood or a combination.

Thus, in these experiments, we investigated the consequences of gut microbiota depletion specifically during adolescence or adulthood and their associated longterm effects on emotional and cognitive behaviours and related-neurochemical measures. The use of ABX allows us to ask specific questions that are unattainable using GF mice. Moreover, as ABX usage can be high in adolescence (e.g. in acne management) ${ }^{28}$ it allows us to have a more translationally relevant manipulation than GF animals.

\section{Experimental procedure}

\section{Animals and experimental design}

Adolescent and adult male $\mathrm{C} 57 \mathrm{Bl} / 6 \mathrm{OlaHsd}$ mice (Envigo, UK) were housed 4 per cage in standard cages. All mice were housed in our animal facility and maintained under a 12-h light/dark cycle. All experiments were conducted in accordance with the European Directive 86/ 609/EEC. Approval by the Animal Experimentation Ethics Committee of University College Cork (2012 \#45) and Health Products Regulatory Authority were obtained before commencement of all experiments. To comply with 3Rs (reduction, refinement and replacement) and animal welfare, the adolescent aspect of the experiment was run simultaneously with another experiment investigating the gut microbiota ${ }^{29}$. The same control group was used in this study.

In order to sufficiently deplete the gut microbiota, a wide-spectrum antibiotic cocktail (ABX) consisting of ampicillin ( $1 \mathrm{~g} / \mathrm{L}$, CAS no. $69-52-3)$, vancomycin $(0.5 \mathrm{~g} / \mathrm{L}$, CAS no. 1404-93-9), ciprofloxacin HCL (0.2 g/L, CAS no. 93107-08-5), imipenem (0.25 g/L, CAS no. 74431-23-5) and metronidazole ( $1 \mathrm{~g} / \mathrm{L}$, CAS no. 443-48-1) was prepared (Fig. 1B) $)^{20,29}$. All substances were purchased from Discovery Fine Chemicals, UK. This antibiotic cocktail has little to no oral bioavailability and was prepared freshly with autoclaved water every second day for 3 weeks (Fröhlich et al., 2016). Control mice received autoclaved water (CTRL). Mice were treated during adolescence (P28-P49) or adulthood (P76-97) and will be referred to as $\mathrm{ABX}_{\text {adolescence }}$ and $\mathrm{ABX}_{\text {adulthood }}$, respectively (see Fig. 1A). Adolescence period in mice was defined as from the day where mice become sexually active (P28) ${ }^{30}$. Behavioural tests commenced 24 days after the final antibiotic exposure. Mice were equally assigned to experimental groups based on body weight to ensure equally distribution among the groups. Each cage represented one treatment. Behavioural tests investigating aspects of anxiety, cognition, social behaviour and fear conditioning were chosen as these behaviours have been shown to be affected by alterations in gut microbiota composition and structure ${ }^{8,31-33}$. Tissue samples were collected $24 \mathrm{~h}$ after the last behavioural test. Body weight was monitored throughout the experiment. The investigators who were involved in sample processing and data analysis were blinded to the groups. All behaviours were assessed by two independent scorers blind to the groups. See supplemental methods for detailed information of all procedures and analysis run in this study.

\section{Elevated plus maze}

The elevated plus maze (EPM) was used to investigate anxiety-like behaviours ${ }^{29}$. Mice were allowed to explore the maze for $5 \mathrm{~min}$; the time spent in the open arms, as 


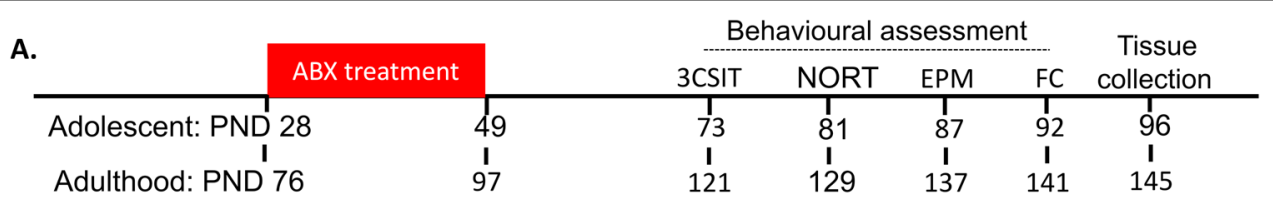

B.

\begin{tabular}{l|l} 
ABX cocktail & $\mathrm{g} / \mathrm{L}$ \\
\hline Ampicilin & 1 \\
\hline Vancomycin & 0.5 \\
\hline Ciprofloxacin HCL & 0.2 \\
\hline Imipenem & 0.25 \\
\hline Metronidazole & 1
\end{tabular}


Fig. 1 Experimental design and body weight performance during the experiment. A Schematic representation of the experimental timeline. Numbers represent the age of the mice at that specific timepoint. B List of drugs used for the antibiotic cocktail. C, D Changes in body weight during adolescence $(\mathbf{C})$ and adulthood (D) over the time course of the experiment. ABX-treated mice show a significant body weight loss on PND10 which is restored afterwards. Mean \pm SEM. ${ }^{*} p<0.05$. Sample size for adolescence: CTRL $n=12$ and ABX $n=10$, adults: CTRL and ABX $n=11$. Two-way repeated measures ANOVA followed by Sidak's post hoc test. PND: postnatal day, CTRL: control, ABX: antibiotic, 3CSIT: three-chambered social interaction test, NORT: novel object recognition task, EPM: elevated plus maze, FC: fear conditioning, Beh: behavioural test.

well as the number of entries into the arms and head dips, were analysed.

\section{Novel object recognition task}

Novel object recognition task (NORT) is a test for short-term memory ${ }^{25}$. Mice were exposed to two identical objects which they could explore for $10 \mathrm{~min}$. One hour later they were exposed to a familiar and a new object. The time they spent exploring the new object was taken as an indication of their memory function.

\section{Three-chamber social approach test}

Sociability and social novelty were investigated using the three-chamber social interaction test $(3 \mathrm{CSIT})^{29}$. The test consisted of three sequential 10-min trials: (1) habituation, (2) sociability, measured as the time the mouse spent in proximity to a conspecific or an object and (3) social novelty preference as measured by the time the mouse spent with an unfamiliar conspecific or a familiar one.

\section{Differential fear-conditioning paradigm}

Fear conditioning (FC) is based on pairing an initially neutral and non-aversive stimulus, an auditory cue or context (conditioned stimulus, CS), with an aversive stimulus, such as a foot shock (unconditioned stimulus, US), which will result in a fear response in the presence of the $\mathrm{CS}^{34}$. The paradigm was run over 4 consecutive days: day 1 (context A): conditioning, day 2 (context A): contextual extinction, day 3 (context B): conditioned extinction in a novel context, day 4 (context B): context recall.

\section{RNA extractions, reverse transcription and quantitative RT-} PCR

Whole amygdala and prefrontal cortex were rapidly gross-dissected on an ice-cooled Petri dish following coordinates described in the "The Mouse Brain in Stereotaxic Coordinates" ${ }^{\mathrm{n}}$ and snap-frozen on dry-ice. For consistency, the same experienced researcher was responsible for all the dissection. These brain regions were chosen as they are major contributor to anxiety and fear learning (amygdala) and proper neuronal communication during development (prefrontal cortex) ${ }^{36-41}$. The list of genes was elaborated together with the experimental design to assess major components modulated by the gut microbiota, such as immune and microglia-related markers, tight-junction proteins and myelin- and stressrelated genes ${ }^{8,29,32,39,40}$. In addition, based on the behavioural phenotype, we included genes associated with anxiety-like behaviour such as the genes involved in the GABAergic and glutamatergic system, NPY system and genes related to neuroplasticity ${ }^{8,26,29,33,36}$. Primer sequences are listed on the Supplementary Table S1. Total RNA was extracted with the mirVana total RNA extraction kit (Ambion, UK) and RNA was reverse transcribed using a high-capacity cDNA reverse transcription kit (Thermo Fisher Scientific, Waltham, USA) in a Gstorm thermocycler (G-storm, Surrey, UK). Real-time PCR was performed on the cDNA samples using SYBR green (SensiFAST ${ }^{\mathrm{TM}}$ SYBR ${ }^{\circledR}$, BioLine, UK) and gene expression levels were analysed on an AB7300 system (Applied Biosystems, Thermo Fisher Scientific, USA). Expression levels were calculated as the average of three replicates for each biological sample from all three groups 
relative to the endogenous control. Fold changes were calculated using the $\Delta \Delta \mathrm{Ct}$ method $^{29}$. The expression of the housekeeper ACTB was not affected by ABX treatment or age.

\section{Caecal microbiota composition (16S rRNA gene sequecing)}

The QIAmp Fast DNA Stool Mini Kit (Qiagen, Sussex, UK) was used for caecal DNA extraction. The procedure was coupled with an initial bead-beating step. Amplification and preparation for sequencing of the V3-V4 hypervariable region of the 16S rRNA gene were done as outlined in the Illumina 16S Metagenomic Sequencing Library Protocol and as previously described ${ }^{29}$. Briefly, microbial genomic DNA was run with each primer (forward primer (5'TCGTCGGCAGCGTCAGATGTGTATAAGAGACAGC CTACGGGNGGCWGCAG-3') and reverse primer (5'GTCTCGTGGGCTCGGAGATGTGTATAAGAGACAG GACTACHVGGGTATCTAATCC-3'). PCR products were purified using the Agencourt AMPure XP system (Beckman Coulter Genomics, UK). Dual indices and Illumina sequencing adapters were attached to $\mathrm{PCR}$ products using the Nextera XT Index Kit (Illumina, USA). PCR products were quantified, normalized and pooled in an equimolar fashion using the Qubit ${ }^{\circledR}$ dsDNA HS Assay Kit (Life Technologies, USA). Following, samples were run on the Agilent Bioanalyser for quality analysis and samples prepared for sequencing following Illumina guidelines. Samples were sequenced on the MiSeq sequencing platform (Clinical Microbiomics, Denmark).

\section{Microbiome bioinformatics processing}

Three-hundred base-pair paired-end reads were prefiltered based on a quality score threshold of $>28$ and trimmed, filtered for quality and chimaeras using the DADA2 library in $\mathrm{R}^{42}$. Samples with fewer than 10,000 reads after trimming and filtering were dropped. Taxonomy was assigned with DADA2 against the SILVA SSURef database release v132. Parameters, as recommended in the DADA2 manual, were adhered to unless mentioned otherwise. ASVs that were only detected as non-zero in 2 or fewer of total samples were excluded.

\section{Statistics}

Power analysis was performed beforehand using the Software G*Power 3.1 to ensure adequate sample size number to detect changes in behaviour and gene expression. Statistical analysis and plotting were conducted using Prism 7 (GraphPad, USA). Data were checked for normality using Shapiro-Wilks normality test while the ROUT method [50] was used to check for outliers. Two-tailed Student's $t$-test (for equal variances) or Welch's $t$-test (for unequal variances) were used for comparison between CTRL and ABX for the EPM, NORT, context and extinction recall in $\mathrm{FC}$, and gene expression. Mann-Whitney nonparametric test was used when dataset failed to have a normal distribution. Twoway repeated measures analysis of variance (ANOVA) was used for body weight, 3CSIT, as well as acquisition and cued extinction in FC. Sidak's multiple comparisons post hoc test was used where applicable. Statistical significance was set at $p<0.05$.

Statistical analysis of microbiota data was performed using the $\mathrm{R}$ software (version 3.6) environment with Rstudio (version 1.1.453). Alpha diversity was calculated using the iNEXT library ${ }^{43}$. Wilcoxon rank sum followed by Bonferroni post hoc tests were used to assess differences in Alpha-diversity scores. For principal component analysis (PCA), permutational multivariate analysis of variance (PERMANOVA) was used to identify relationships of significance between variables using the adonis() function from the vegan library on Aitchison distance matrices calculated with the ALDEx2 library ${ }^{44}$. A pairwise implementation of the ALDEx2 function aldex.t.test() was also used to calculate pairwise differential abundance, using the Bonferroni procedure as a post hoc. In the case of microbiome analysis, the Benjamini-Hochberg procedure was used to account for false discovery rate due to multiple comparisons, a $q$-value of 0.1 was deemed significant.

\section{Results}

Antibiotic treatment in adolescence affects body weight

Two-way repeated measures ANOVA showed an overall effect of $A B X$ treatment during adolescence on body weight (interaction $\mathrm{F}(3,48)=3.84, p=0.015$, treatment $\mathrm{F}(1,16)=$ $7.18, p=0.017$ ), whereas such an effect was not seen following treatment with $\mathrm{ABX}_{\text {adulthood }}$ (interaction $\mathrm{F}(3,60)=$ 12.56, $p<0.001$, treatment $\mathrm{F}(1,20)=0.11, p=0.75$; Fig. $1 \mathrm{C}$, D). Sidak's multiple comparison, however, revealed that body weight was significantly lower in the $\mathrm{ABX}$ treatment in both $\mathrm{ABX}_{\text {adolescence }}$ and $\mathrm{ABX}_{\text {adulthood }}$ mice on day 10 of the treatment ( $p=0.003$ and $p=0.05$, respectively). On day 10 , the body weight loss in ABX-treated mice compared to the CTRL was $14 \%$ and $12 \%$ for adolescent and adults mice, respectively.

\section{Microbial diversity was only affected by ABX treatment during adolescence}

To investigate possible long-lasting effects of $A B X$ treatment during adolescence and adulthood on the gut microbiota, microbiota was sequenced from caecal contents 49 days after termination of the treatment. Overall, sequencing demonstrated that the gut microbiota was shifted only in $A B X_{\text {adolescence }}$ mice where $A B X$ treatment had reduced relative abundance and diversity compared to CTRL (Fig. 2A-C). With regards to beta-diversity, PERMANOVA identified significant differences between CTRL and ABX treatment in adolescent mice $(p<0.001$, 
A oCTRL Adolescence o CTRL Adulthood
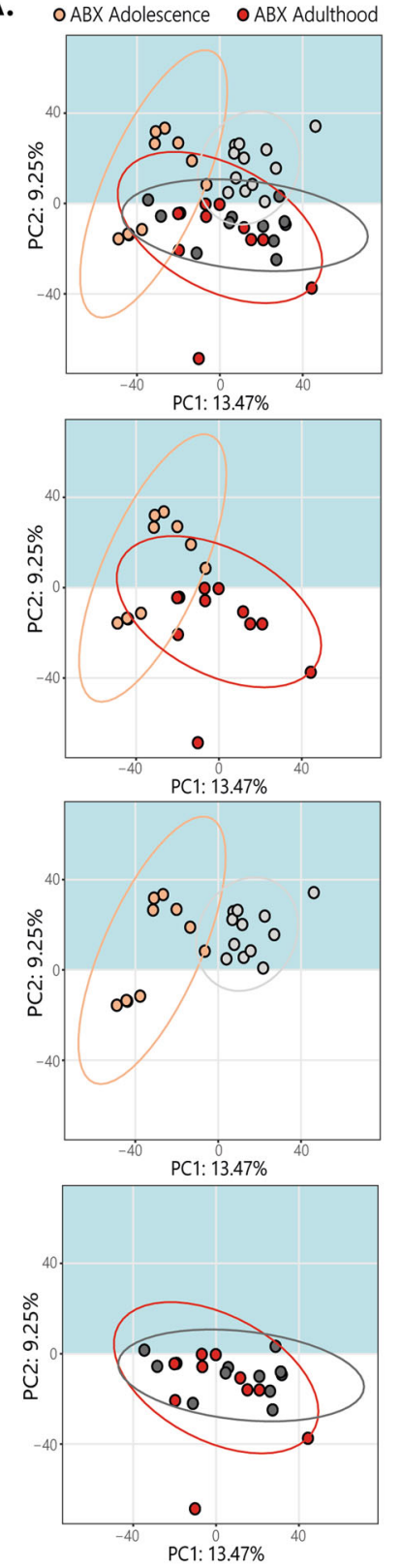

B.

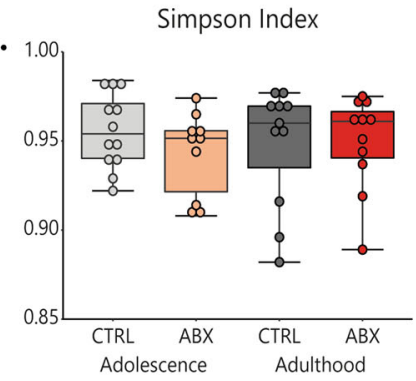

C.
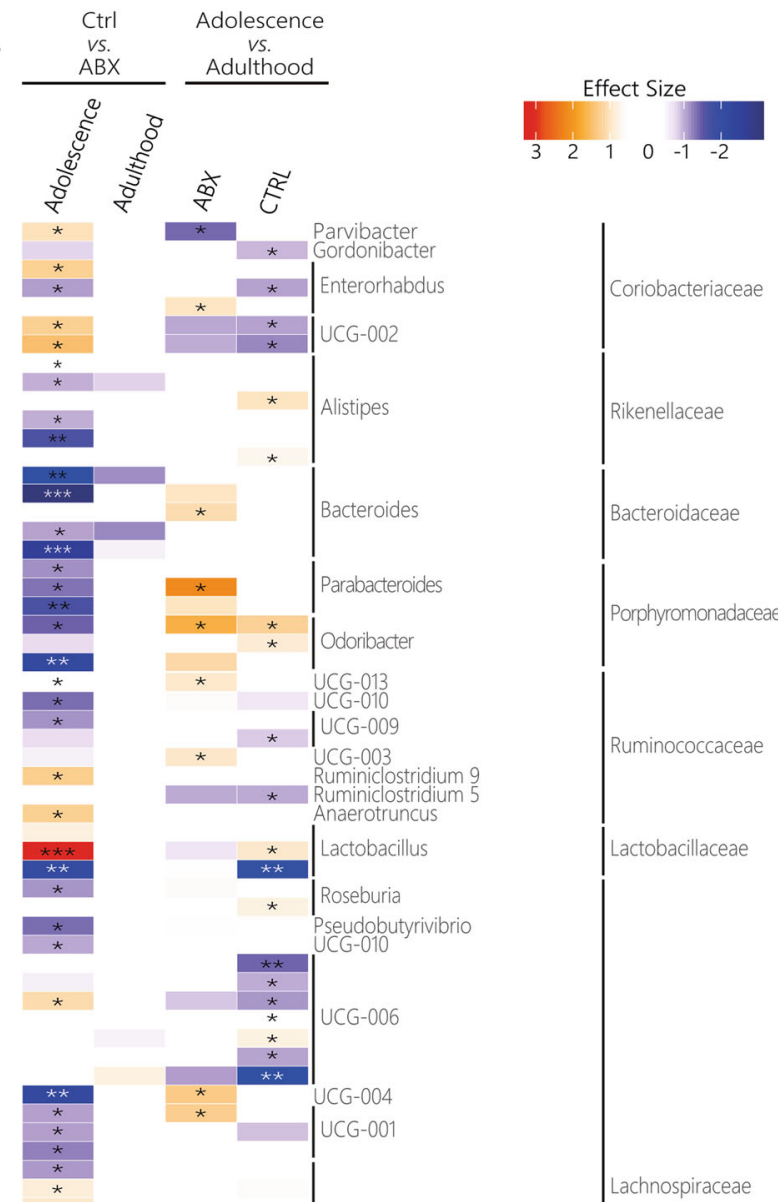

UCG-003
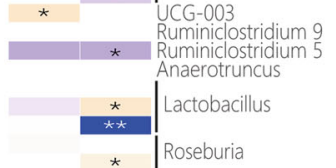

* $\quad$ Roseburia
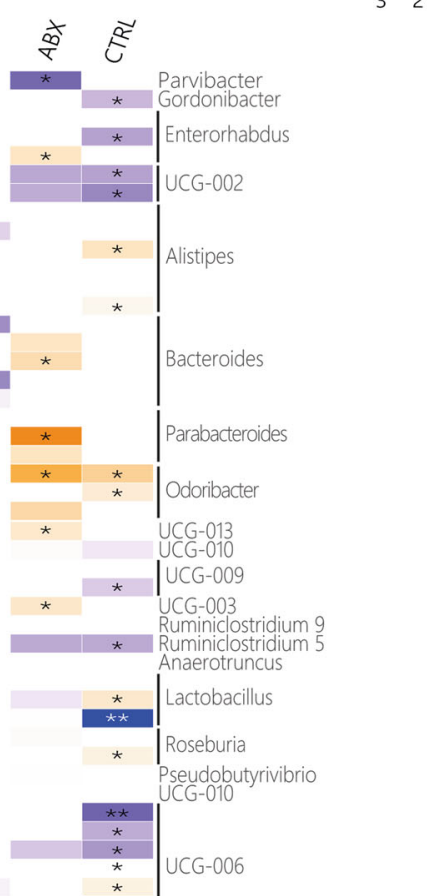

$\begin{array}{llll}1 & 0 & -1 & -2\end{array}$

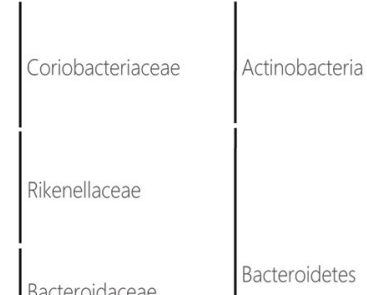

Porphyromonadaceae



Bacteroidacea


Lachnospiraceae

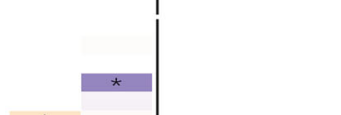

Lachnospiraceae

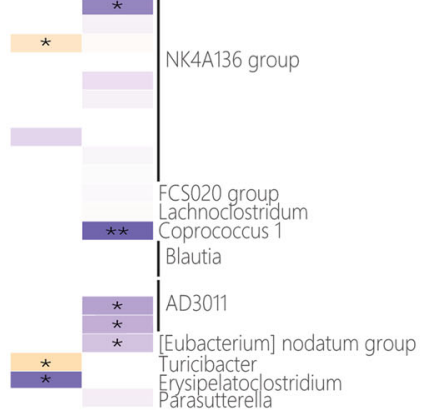

Family XIII

Erysipelotrihaceae

Alcaligenaceae 
(see figure on previous page)

Fig. 2 Long-lasting changes in caecal microbiota composition following ABX intervention during adolescence. A PCA blots depicting differences in beta-diversity between mice treated with $A B X$ and their respective controls. While beta-diversity was affected by $A B X_{\text {adolescence }}$ treatment no such effects were seen following $A B X_{\text {adulthood }}$ treatment. B alpha-diversity indices: Chao 1, Shannon and Simpson. Changes in the Chao 1 and Simpson indices are observed following treatment with $\mathrm{ABX}$ during adolescence but not after treatment in adulthood. $\mathbf{C}$ Heat map representing relative abundance of ASVs. Significant differences were observed between control mice and ABX-treated mice during adolescence but not when treated during adulthood. Some differences in ASVs were observed when comparing both ABX treatments and controls with one another. The latter does not explain the effect on relative abundance of $A B X$ treatment during adolescence. Mean $\pm S E M .{ }^{*} p<0.05$. Sample size for adolescence: $C T R L$ $n=12$ and $A B X n=10$; for adults: CTRL and $A B X n=11$. A Permutational multivariate analysis of variance (PERMANOVA), followed by pairwise PERMANOVA post hoc Benjamini-Hochberg, B Wilcoxon rank-sum test, post hoc Bonferroni, ${ }^{*} p<0.05$, C Mann-Whitney $U$ test post hoc Benjamini-Hochberg, Benjamini-Hochberg false discovery rate (FDR) $q<0.2$. Asterisks in the heat map represent the following $q$ values: ${ }^{*} p<0.1,{ }^{* *} p$ $<0.01,{ }^{* *} p<0.001$

$R^{2}=0.193$, padj $=0.004 ;$ Fig. $\left.2 \mathrm{~A}\right)$, but no such change was observed in $\mathrm{ABX}_{\text {adulthood }}\left(p=0.029, R^{2}=0.071, \operatorname{padj}=\right.$ 0.174; Fig. 2A).

Alpha diversity, which describes the diversity of species within a community, was measured by calculating indices for richness and evenness based on amplicon sequence variants (ASV) level. Details of the statistics for each ASV can be found on the Supplementary Table S2. Figure 2B shows reduced numbers of bacterial species between CTRL and ABX treatment in adolescent mice (Chao1 Index; pairwise comparison using Wilcoxon rank-sum test, $p<0.001)$ while no changes were observed in $\mathrm{ABX}_{\text {adulthood }}$ mice. Moreover, a significant decrease in evenness across ASVs was observed in $\mathrm{ABX}_{\text {adolescence }}$ mice (Shannon Index; pairwise comparison using Wilcoxon rank-sum test, $p<0.001$ ), indicating that treatment with $\mathrm{ABX}$ during adolescence disrupted the uniformity of the population size of each of the species present. No effects of treatment during adolescence or adulthood were observed for the Simpson's index.

$A B X$ treatment only resulted in significant longlasting changes in relative abundance of adolescent mice. $A B X_{\text {adolescence }}$ mice showed reduced relative abundance of most of the strains affected. At the phylum level, Firmicutes and Bacteriodetes were most


Actinobacteria had a marginal shift that was restricted to the genus Coriaobacteriaceae UCG002 and Parvibacter, found increased, and Enterorhabdus, with mixed result. Relative abundance of all the affected genera in the phylum Bacteriodetes was decreased, with the strongest reduction in the genera Bacteroides, while Parabacteroides, Odoribacter and Alistipes had less strong effect. Members of the Firmicutes phylum were differentially in $\mathrm{ABX}_{\text {adolescence. In the Lachnospiraceae }}$ family, relative abundance of all affected ASV of the genera Rosburia, UCG-001, UCG-004, UCG-010, FCSO20 group and Pseudobutyrivibrio were decreased, whereas at UCG-006, NK4A136 group, Lachnoclostridium and Blautia showed increased relative abundance in $\mathrm{ABX}_{\text {adolescence. }}$ Of the family Ruminococcaceae, relative abundance of the genera UCG-003, UCG-009, UCG-010 and UCG-013 was decreased while the genera Ruminiclostridium 5, Ruminiclostridium 9 and Anaerotruncus was significantly increased. Similarly, one ASV of the genus Lactobacillus was decreased while another ASV of this genus was strongly increased following treatment with $\mathrm{ABX}$. The relative abundance of all affected ASVs of the family Family XIII and Eryspelotrihaceae was decreased. The phylum Proteobacteria had just one family changed after $A B X$ treatment, where Parasutterella was found decreased.

In $\mathrm{ABX}_{\text {adulthood }}$, no significant effect on relative abundance was observed. However, differences in relative abundance were observed in CTRL or ABX groups between adolescent and adults mice. As expected, CTRL mice from adolescence and adults have different microbiota composition and diversity (Fig. 2A-C). When comparing $\mathrm{ABX}$ treatment of adolescent versus adult mice, relative abundance of most of the ASVs is increased in $\mathrm{ABX}_{\text {adulthood }}$ mice in comparison to $A B X_{\text {adolescence }}$ treated mice and vice versa (e.g. ASVs of the genera Parabacteroides and Odoribacter). This is in line with the decrease observed in the comparison of relative abundance of $\mathrm{ABX}_{\text {adolescence }}$ mice and their controls. Similarly, the differences seen between the controls of either treatment is not in contradiction with the differences observed in relative abundance of $\mathrm{ABX}_{\text {adolescence }}$ mice in comparison to their controls. The differences in relative abundance between controls mostly occurred in ASVs that are not affected in the $\mathrm{ABX}_{\text {adolescence, }} 14$ out of the $25 \mathrm{ASVs}$ that were different between the controls were not significantly affected by $\mathrm{ABX}_{\text {adolescence }}$ treatment (e.g. ASVs of the genus Alistipedis).

\section{Treatment with antibiotics strongly affected anxiety-like behaviour, with a more pronounced effect in mice treated during adolescence}

To test the effects of $\mathrm{ABX}$ treatment on anxiety-like behaviour, mice were tested in the EPM. Mice that underwent antibiotic treatment showed increased anxiety-like behaviour. Unpaired $t$-test with Welch's correction showed 
that the percentage of time into the open arms, as well as the number of head dips $(t=2.846, p=0.01$ and $t=2.909$, $p=0.01$, respectively; Fig. 3A, B), were affected significantly in $\mathrm{ABX}_{\text {adolescence }}$ mice. Student $t$-test showed that the percentage of entries into the open arms also was affected significantly in $\mathrm{ABX}_{\text {adolescence }}$ mice $(t=2.529, p=0.02$; Fig. $3 C)$. Welch's $t$-test revealed no statistical significance in the percentage of entries into the open arm in $\mathrm{ABX}_{\text {adulthood }}$ mice $(t=1.946, p=0.06$; Fig. 3K). Mann-Whitney nonparametric test found that the percentage of time into the open arms as well as head dips were no difference in $\mathrm{ABX}_{\text {adulthood }}$ compared to the controls $(U=38, p=0.148$ and $U=38$, $p=0.145$, respectively; Fig. 3I, J). Entries into the closed arm did not differ between treatment groups (adolescence: $t=$ 0.419, $p=0.68$; adulthood: $t=0.449, p=0.65$; Fig. 3D, L), indicating that $\mathrm{ABX}$ treatment did not affect locomotor activity.

\section{Sociability and memory processes were not affected by antibiotic treatment in adolescence or adulthood}

As social behaviour and cognition have been shown to be impacted by alterations in gut microbiota composition, we investigated the effects of $\mathrm{ABX}$ treatment on measures in 3CSIT and NORT. ABX treatment during adolescence or adulthood had no effects on sociability or social novelty. Two-way repeated measures ANOVA found preferential interaction for a stranger mouse (S1) over an innate object $(\mathrm{O})(\mathrm{S} 1 \times \mathrm{O}: \mathrm{F}(1,17)=52.92, p<0.001$, treatment: $\mathrm{F}(1,17)=1.76, p=0.20$ for adolescence; $\mathrm{S} 1 \times$ O: $\mathrm{F}(1,20)=60.54, p<0.001$, treatment: $\mathrm{F}(1,20)=0.013$, $p=0.90$ for adulthood, supplementary Fig. 1B, E, respectively). Sidak's multiple comparison post hoc test found that CTRL and ABX from both adolescence and adult groups preferred S1 over $\mathrm{O}(p<0.0001)$. On social novelty, two-way repeated measures ANOVA found that only the adolescence group has a preference between stranger and familiar mouse $(\mathrm{S} 1 \times \mathrm{S} 2: \mathrm{F}(1,17)=13.96, p<$ 0.01 , treatment: $\mathrm{F}(1,17)=3.03, p=0.09$ for adolescence; $\mathrm{S} 1 \times \mathrm{S} 2: \quad \mathrm{F}(1,20)=2.74, \quad p=0.11$, treatment: $\mathrm{F}(1,20)=$ $0.61, p=0.44$ for adulthood, supplementary Fig. 1C, F, respectively). Sidak's multiple comparison post hoc test found that ABX-treated adolescent preferred S1 over S2 $(p<0.001)$ but not CTRL-treated mice $(p=0.10)$.

In the NORT, neither $\mathrm{ABX}_{\text {adolescence }}$ or $\mathrm{ABX}_{\text {adulthood }}$ exhibited differences in the ability to recognize a distinct object $1 \mathrm{~h}$ after the training session, expressed by the time exploring the novel object (adolescence: $t=1.233, \mathrm{df}=$ 16.47, $p=0.23$; adulthood: $t=0.5288, \mathrm{df}=16.77, p=$ 0.60, Supplementary Fig. 2A, D).

\section{Fear learning is only affected in animals treated with antibiotics during adolescence}

Fear conditioning aimed to investigate different fear responses, such as fear learning, contextual fear and cued extinction (Fig. 3E-H, M-P). All experimental groups learned the task as indicated by increasing freezing behaviour during acquisition (two-way repeated measures ANOVA; CS presentation, adolescence: $\mathrm{F}(4,68)=38.11$, $p<0.001$; adulthood: $\mathrm{F}(4,80)=27.36, p<0.001$; Fig. 3E, $\mathrm{M})$. Only $\mathrm{ABX}_{\text {adolescence }}$ mice significantly increased freezing behaviour compared to the control group during fear acquisition, while in adults, $\mathrm{ABX}$ did not affect fear acquisition (two-way repeated measures ANOVA; treatment, adolescence: $\mathrm{F}(1,17)=4.48, p=0.04$; adulthood: $\mathrm{F}$ $(1,20)=0.17, p=0.67)$. Sidak's multiple comparison post hoc test did not find a significance between CTRL and $\mathrm{ABX}$ in any timepoint in both adolescence and adults mice. On day 2, contextual fear was assessed. Unpaired $t$ test with Welch's correction revealed that the treatment did not affect freezing behaviour in adolescent mice $(t=$ $1.70, p=0.10$; Fig. 3F). Student $t$-test revealed the same in adult mice $(t=0.73, p=0.47$; Fig. $3 N)$. In the cued extinction session (day 3 ), the response to the CS (paired sound) was analysed. All experimental groups reduced their freezing response over the course of CS presentation (CS presentation, two-way repeated measures ANOVA adolescence: $\mathrm{F}(4,68)=18.03, \quad p<0.001$, adulthood: $\mathrm{F}$ $(4,80)=15.15, p<0.001$; Fig. 3G, O), suggesting that all experimental groups were able to extinguish cued fear. Fear extinction was not affected by treatment (two-way repeated measures ANOVA; adolescence: $\mathrm{F}(1,17)=0.095$, $p=0.76$, adulthood: $\mathrm{F}(1,20)=0.11, p=0.74)$. Pre and post CS freezing were similar in ABX-treated mice compared to their controls (Welch's $t$-test; adolescence; pre: $t$ $=0.36, p=0.72$; post: $t=0.61, p=0.54$; adulthood; pre: $t$ $=0.22, p=0.82$; post: $t=0.15, p=0.88$ ), indicating the absence of generalized fear. Fear recall was tested on day four and no difference was observed (Welch's $t$-test; adolescence: $t=0.86, p=0.39$, adulthood $t=0.93, p=$ 0.36; Fig. $3 \mathrm{H}, \mathrm{P})$. Taken together, this data show that transient depletion of the gut microbiota has little potential to long-lasting affect fear response.

\section{Amygdala gene expression is more strongly affected by treatment during adolescence than adulthood}

Many studies have demonstrated a link between the gut microbiota and gene expression in the amygdala ${ }^{39,45}$. In amygdala homogenates, mRNA levels revealed a stronger effect of $\mathrm{ABX}_{\text {adolescence }}$ than $\mathrm{ABX}_{\text {adulthood }}$ treatment (Fig. 4 and Table 1). Immune markers were heavily affected by ABX treatment during adolescence and adulthood (Fig. 4A, E). For instance, elevated levels of Fcgr2b (Fc fragment of IgG receptor IIb) gene were observed in both $A B X_{\text {adolescence }}$ and $\mathrm{ABX}_{\text {adulthood }}$ mice. The toll-like receptor 4 gene (Tlr4) was oppositely affected by $\mathrm{ABX}$ treatment with elevated gene expression following $\mathrm{ABX}_{\text {adolescence }}$ treatment and reduced expression after $\mathrm{ABX}_{\text {adulthood }}$ treatment. In contrast, interleukin 1b (Illb) was elevated following 


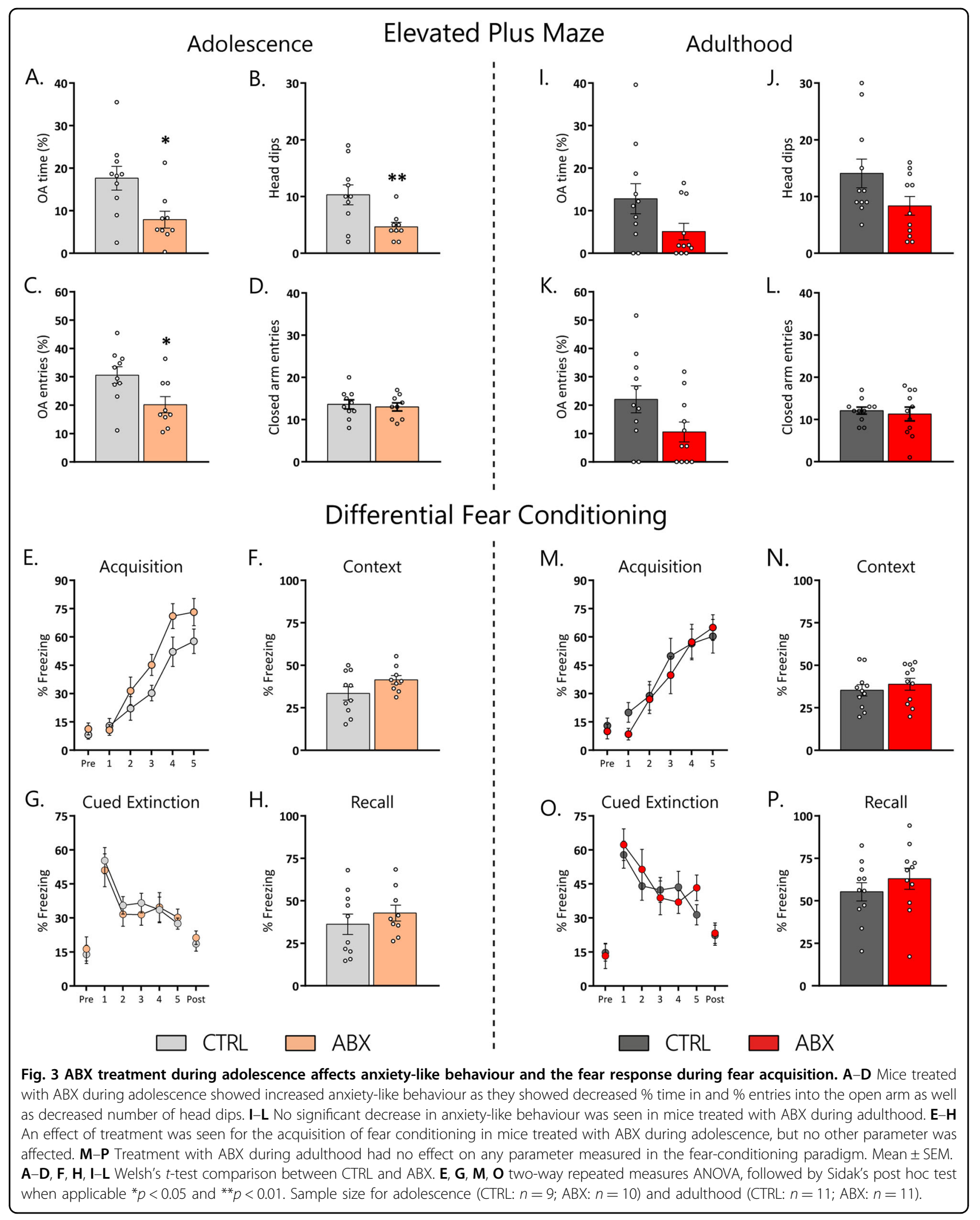









\begin{abstract}
(see figure on previous page)
Fig. 4 Amygdalar gene expression is more drastically changed following ABX treatment during adolescence. A, B While genes related to neuroimmunity and microglia are similarly affected in $A B X_{\text {adolescence }}$ and $A B X_{\text {adulthood }}$ mice, gene expression genes involved in $\mathbf{C}, \mathbf{D}$ neurotransmission, $\mathbf{E}, \mathbf{F}$ neuroplasticity, short-chain fatty acids and tight-junction proteins are more drastically affected in $\mathrm{ABX}_{\text {adolescence }}$ mice. Mean \pm SEM. Unpaired $t$-test with Welch's correction comparing vehicle and antibiotic treatment for each gene. ${ }^{*} p<0.05,{ }^{* *} p<0.01$ and ${ }^{* * *} p<0.001$. Sample size for adolescence: CTRL and ABX $n=8$ (II10, II1b, Gabra2, Gabbr1, Npy1r, Psd95 and Tjp 1), CTRL $n=8$ and ABX $n=9$ (Fcgr2b, Cx3cr1, Rac2, C3, Clqa, Grm5, Chr1r, Npy, Syp, Ffar1, Slc5a8, Slc16a1, Ocln), CTRL $n=9$ and ABX $n=8$ (T/r4), CTRL and ABX $n=9$ (Nr3c1). Sample size for adults: CTRL and ABX $n=9$ (Fcgr2b, II1b, CX3cr1, Rac2, C3, Gabra2, Gabbr1, Grm5, Crh1r, Npy1r, Psd95, Syp, Ffar1, Slc5a8, Ocln, Tjp1), CTRL $n=10$ and ABX $n=9$ (II10, Trr4, $\mathrm{Nr3C1}$, Slc16a1), CRTL $n=9$ and ABX $n=8$ (C1qa, Npy). SCFA: short-chain fatty acid, C1qa: complement C1q subunit A, C3: complement component 3, Crh 1r: corticotropin-releasing hormone receptor 1, Cx3cr1: chemokine receptor 1, Fcgr2b: Fc fragment of IgG receptor Ilb, Ffarl: free fatty acid receptor 1, Gabrb1: gamma-aminobutyric acid type B receptor subunit 1, Gabra2: gamma-aminobutyric acid type A receptor alpha2 subunit, Grm5: glutamate metabotropic receptor 5, II1b: interleukin 1b, II10: interleukin 10, Npy: neuropeptide Y, Npy1r: neuropeptide Y receptor Y1, Nr3c1: glucocorticoid receptor, OcIn: occludin, PSD95: postsynaptic density protein 95, Rac2: Ras-related C3 botulinum toxin substrate 2, S/c5a8: solute carrier family 5 member 8, S/c16a1: solute carrier family 16 member 1, Syp: Synaptophysin, Tjp 1: tight-junction protein 1, T/r4: toll-like receptor 4.
\end{abstract}

$\mathrm{ABX}_{\text {adulthood }}$ treatment but not following $\mathrm{ABX}_{\text {adolescence }}$ treatment, while the microglia-related gene C1qa (encodes the A-chain polypeptide of serum complement subcomponent $\mathrm{C} 1 \mathrm{q}$ ) and $\mathrm{C} x 3 \mathrm{cr} 1$ (interleukin 8 receptor, alpha) was only upregulated in $\mathrm{ABX}_{\text {adolescence }}$ mice. Rac2 (Rho GTPase Rac2), which regulates phagocytosis was upregulated in both experimental groups. The complement component 3 (C3) which is also involved in the regulation of phagocytosis, was only upregulated in $\mathrm{ABX}_{\text {adulthood }}$ mice. Classical and peptidergic neurotransmission was strongly affected by $\mathrm{ABX}_{\text {adolescence }}$ treatment but remained mostly unaltered in $\mathrm{ABX}_{\text {adulthood }}$ mice (Fig. 4B, F). The levels of gamma-aminobutyric acid receptor subunit alpha-2a (Gabra2) and beta-1b (Gabrb1), the metabotropic glutamate receptor subunit 5 (Grm5), neuropeptide Y (NPY) and its $\mathrm{Y} 1$ receptor $(N p y 1 r)$ and the glucocorticoid receptor $\left(\mathrm{Nr} 3 \mathrm{cl}\right.$ ) were only increased in $\mathrm{ABX}_{\text {adolescence }}$ mice. The corticotropin-releasing hormone receptor subunit 1 (Crh1r) was the only gene affected in $\mathrm{ABX}_{\text {adulthood }}$ mice, that was not altered by $\mathrm{ABX}_{\text {adolescence }}$ treatment. Neuroplasticity genes were differently affected by $\mathrm{ABX}_{\text {adolescence }}$ and $\mathrm{ABX}_{\text {adulthood }}$ (Fig. 4C, G). While the levels of postsynaptic density protein 95 (Psd95) and synaptophysin (Syp) were increased in $\mathrm{ABX}_{\text {adolescence }}$ mice, these genes were downregulated following $\mathrm{ABX}_{\text {adulthood }}$ treatment. Interestingly, genes associated with short-chain fatty acids (SCFAs), the solute carrier subtypes (Slc5a8 and Slc16a1) were only elevated in $\mathrm{ABX}_{\text {adolescence }}$ mice. Similarly, the gene expression of tight-junction genes, such as occludin (Ocln) and tight-junction protein 1 (Tjp1) were only increased in $\mathrm{ABX}_{\text {adolescence }}$ mice. Taken together, although more genes are affected in $\mathrm{ABX}_{\text {adolescence, immune-related genes are }}$ similarly affected by both ABX treatments.

Finally, we analysed myelination-related genes in the prefrontal cortex, which have been suggested to be modulated by the gut microbiota ${ }^{20,46}$. Therefore, we analysed gene expression of myelin regulatory factor (Myrf), proteolipid protein 1 (Plp1) and the transcription factor SOX-10 (Sox10). $\mathrm{ABX}_{\text {adulthood }}$ treatment resulted in increased gene expression of Myrf and Sox10 while $\mathrm{ABX}_{\text {adolescence }}$ showed no effects (Supplementary Fig. 2A, B).

\section{Discussion}

Adolescence is a particularly vulnerable time for the onset of psychopathology. Understanding what factors mediate such susceptibility is an important area in biological psychiatry research. In the present studies, we assessed the long-term effects of transient gut microbiota depletion using an established $A B X$ cocktail in mice during adolescence or adulthood. $\mathrm{ABX}_{\text {adolescence }}$ had long-lasting effects on composition and structure of the gut microbiota and anxiety-like behaviour, whereas $\mathrm{ABX}_{\text {adulthood }}$ had no effects. This long-lasting shift in the gut microbiota when depleted during adolescence highlights the vulnerability of the gut microbiota during development and may be responsible for the anxiety-like phenotype and pronounced changes in gene expression within the amygdala observed in $\mathrm{ABX}_{\text {adolescence }}$ mice. To our knowledge, this is the first study demonstrating that the long-lasting effects on gut microbiota only occur when treated during a critical developmental period.

In mammals, the initial microbiota is mainly acquired at birth and later shaped during the developmental period by genetics and environmental factors until become increasingly stable and resistant during adulthood ${ }^{2,19,21}$. Adolescence is a period when major physiological transformation occurs. Sexual hormones such as testosterone and oestrogen are peaking during puberty and it is known to affect the composition of the gut microbiota ${ }^{2,47,48}$. Comparatively, the gut microbiota during the adolescence is less diverse, with a lower Firmicutes/Bacteriodetes ratio than adults ${ }^{49}$. Consequently, environmental stressors such as poor diet, antibiotics, exercise among other can impair the gut microbiota differently in adolescence and adults, with the later demonstrating higher resilience to harmful events compared to adolescence or elderly.

Use of antibiotics strongly affect the Firmicutes/Bacteriodetes ratio ${ }^{16,18-20,25-27,50-52}$ and could be determinant to 
Table 1 Statistical values of the gene expression in the amygdala, prefrontal cortex following ABX intervention during adolescence and adult.

\begin{tabular}{|c|c|c|c|c|}
\hline \multirow[b]{2}{*}{ Gene } & \multicolumn{2}{|l|}{ Adolescent } & \multicolumn{2}{|l|}{ Adult } \\
\hline & $t, \mathrm{df} / \mathrm{U}, t_{1 / 2}$ & $p$ & $t, \mathrm{df} / \mathrm{U}, t_{1 / 2}$ & $p$ \\
\hline \multicolumn{5}{|c|}{ Amygdala } \\
\hline Fcgr2b & $t_{(\mathrm{W})}=4.732, \mathrm{df}=13.59$ & $<0.001$ & $t_{(\mathrm{W})}=2.167, \mathrm{df}=15.8$ & 0.05 \\
\hline Clqa & $t_{(\mathrm{W})}=3.938, \mathrm{df}=13.16$ & $<0.001$ & $t_{(\mathrm{m})}=0.765, \mathrm{df}=14.37$ & 0.46 \\
\hline$T / r 4$ & $t_{(\mathrm{W})}=5.658, \mathrm{df}=13.92$ & $<0.001$ & $U=10 ; t_{1}=135, t_{2}=55$ & 0.003 \\
\hline 1110 & $t_{(\mathrm{W})}=0.097, \mathrm{df}=11.27$ & 0.92 & $U=32 ; t_{1}=87, t_{2}=103$ & 0.31 \\
\hline$\| 1 B$ & $t_{(\mathrm{W})}=0.079, \mathrm{df}=12.96$ & 0.94 & $t_{(\mathrm{m})}=2.356, \mathrm{df}=14.15$ & 0.03 \\
\hline $\mathrm{C} \times 3 \mathrm{cr} 1$ & $U=1 ; t_{1}=37, t_{2}=116$ & $<0.001$ & $t_{(m)}=0.400, d f=15.72$ & 0.69 \\
\hline Rac2 & $t_{(\mathrm{W})}=5.679, \mathrm{df}=9.484$ & $<0.001$ & $t_{(\mathrm{W})}=6.379, \mathrm{df}=11.64$ & $<0.001$ \\
\hline C3 & $t_{(\mathrm{W})}=1.643, \mathrm{df}=14.89$ & 0.12 & $t_{(m)}=2.951, d f=15.29$ & 0.01 \\
\hline Gabra2 & $t_{(\mathrm{W})}=5.427, \mathrm{df}=11.06$ & $<0.001$ & $U=20 ; t_{1}=65, t_{2}=106$ & 0.07 \\
\hline Gabrb1 & $t_{(\mathrm{W})}=3.42, \mathrm{df}=13.44$ & $<0.001$ & $U=37 ; t_{1}=82, t_{2}=89$ & 0.79 \\
\hline Grm5 & $t_{(\mathrm{W})}=8.899, \mathrm{df}=11.21$ & $<0.001$ & $t_{(\mathrm{m})}=0.409, \mathrm{df}=13.89$ & 0.69 \\
\hline Psd95 & $U=0 ; t_{1}=36, t_{2}=100$ & $<0.001$ & $t_{(\mathrm{W})}=3.724, \mathrm{df}=11.06$ & $<0.001$ \\
\hline Syp & $t_{(\mathrm{W})}=4.609, \mathrm{df}=13.46$ & $<0.001$ & $t_{(\mathrm{W})}=4.88, \mathrm{df}=13.49$ & $<0.001$ \\
\hline Npy & $t_{(\mathrm{W})}=5.087, \mathrm{df}=12.06$ & $<0.001$ & $t_{(\mathrm{W})}=0.865, \mathrm{df}=10.51$ & 0.41 \\
\hline $\mathrm{Nr3Cl}$ & $t=4.087, \mathrm{df}=16$ & $<0.001$ & $U=39 ; t_{1}=106, t_{2}=84$ & 0.64 \\
\hline Npy1r & $t_{(\mathrm{W})}=3.842, \mathrm{df}=8.787$ & $<0.001$ & $t_{(\mathrm{w})}=0.992, \mathrm{df}=11.31$ & 0.34 \\
\hline Chr1r & $t=0.2401, \mathrm{df}=15$ & 0.81 & $U=8 ; t_{1}=118, t_{2}=53$ & 0.02 \\
\hline FFAR1 & $t_{(\mathrm{m})}=26 ; t_{1}=62, t_{2}=91$ & 0.19 & $t_{(\mathrm{m})}=1.736, \mathrm{df}=12.16$ & 0.11 \\
\hline$S / c 5 a 8$ & $U=4 ; t_{1}=40, t_{2}=113$ & $<0.001$ & $U=30 ; t_{1}=75, t_{2}=96$ & 0.38 \\
\hline Slc16ar & $t_{(\mathrm{W})}=6.466, \mathrm{df}=12.82$ & $<0.001$ & $U=21 ; t_{1}=76, t_{2}=114$ & 0.05 \\
\hline Ocln & $t_{(\mathrm{W})}=3.641, \mathrm{df}=14.88$ & $<0.001$ & 0.34 & \\
\hline Tjp1 & $t_{(\mathrm{w})}=3.759, \mathrm{df}=12.05$ & $<0.001$ & $t=1.171, \mathrm{df}=16$ & 0.26 \\
\hline \multicolumn{5}{|c|}{ Prefrontal cortex } \\
\hline Myrf & $U=36 ; t_{1}=91, t_{2}=99$ & 0.49 & $t_{(\mathrm{W})}=7.641, \mathrm{df}=14.12$ & $<0.001$ \\
\hline Plp1 & $t_{(\mathrm{W})}=0.489, \mathrm{df}=14.92$ & 0.63 & $U=45 ; t_{1}=111, t_{2}=120$ & 0.49 \\
\hline Sox 10 & $t_{(\mathrm{W})}=0.762, \mathrm{df}=16.89$ & 0.46 & $t=4.934, \mathrm{df}=19$ & $<0.001$ \\
\hline
\end{tabular}

Mean \pm SEM. Unpaired $t$-test with or without Welch's correction or Mann-Whitney whenever the dataset did not follow normal distribution.

$t$ Student $t$-test, $t_{(\mathrm{W})}$ Student $t$-test with Welch's correction, $d f$ degree of freedom, $U$ Mann-Whitney test, $t_{1 / 2}$ sum of ranks of CTRL and ABX, C1qa complement C1q subunit A, C3 complement component 3, Crh1r corticotropin-releasing hormone receptor 1, Cx3cr1 chemokine receptor 1, Fcgr2b Fc fragment of lgG receptor Ilb, Ffar 1 free fatty acid receptor 1, Gabrb1 gamma-aminobutyric acid type B receptor subunit 1, Gabra2 gamma-aminobutyric acid type A receptor alpha2 subunit, Grm5 glutamate metabotropic receptor 5, I/1b Interleukin 1b, I/10 interleukin 10, Myrf myelin regulatory factor, Npy neuropeptide Y, Npy $1 \mathrm{r}$ neuropeptide Y receptor Y1, Nr3c1 glucocorticoid receptor, Ocln occludin, Plp1 proteolipid protein 1, Psd95 postsynaptic density protein 95 , Rac2 Ras-related C3 botulinum toxin substrate 2, S/c5a8 solute carrier family 5 member 8, Slc16a1 solute carrier family 16 member 1, Sox 10 SRY-box transcription factor 10, Syp synaptophysin, Tjp1 tight-junction protein 1, TIr4 tolllike receptor 4.

facilitate changes in brain function and increase susceptibility to anxiety levels ${ }^{33,49,52}$. Evidence has suggested that changes in the microbiota composition can also regulate sexual hormone production, which would have a larger impact during the adolescence ${ }^{4,47,48}$. Thus, disrupting normal growth trajectory of the gut microbiota and its inability to bounce back to its original composition following the treatment may have contributed to the longlasting effect after ABX treatment in adolescent ${ }^{53,54}$.

Many studies have described that 1-5 weeks of $A B X$ treatment is enough to deplete the gut microbiota and influence the expression of genes and behaviour ${ }^{8,20,25,26}$. By depleting the gut microbiota consistently over most of the adolescence period, we observed a statistically 
significant increase in anxiety-like behaviour in the EPM only in $\mathrm{ABX}_{\text {adolescence }}$ mice, in line with the changes in microbiota composition and structure. The role of gut microbiota depletion in anxiety, however, is controversial with studies describing a reduction of anxiety-like behaviours $^{25,55}$, no effect ${ }^{20,26}$ or an anxiolytic effect following microbiota depletion ${ }^{16}$. Firmicutes, Actinobacteria and Bacteroides are known to affect anxiety levels, with lower abundance being associated with elevated anxiety-like behaviour in rodents and humans ${ }^{36,56-58}$. The stronger effect observed in $\mathrm{ABX}_{\text {adolescence }}$ mice over adults may also have been influenced by sex hormones peaking during adolescence. Preclinical and clinical studies in males suggest that testosterone yields protective benefits against anxiety. Moreover, high levels of testosterone during the adolescent period is known to influence the commensal bacteria Firmicutes/Bacteroides ratio $^{4,47,48}$, suggesting that interaction of $\mathrm{ABX}$ with testosterone during adolescence could have an additive effect on the gut microbiota composition.

In addition, it has been found that administration of probiotics containing species of the genus Lactobacillus and Bifidobacterium correlates with the expression of Gabrb1 within the amygdala, an effect that has been shown to be influenced by vagal communication ${ }^{36,59}$. Interestingly, Lactobacilli species appeared mainly increased and Bifidobacterium was unchanged in $\mathrm{ABX}_{\text {adolescence. }}$ Beneficial bacterial species such as Lactobacillus and Bifidobacterium are usually found decreased after $A B X$ treatment in rodents ${ }^{60}$ and elevated Lactobacillus/Bifidobacterium levels in the gut microbiota or use of probiotic preparations have an anxiolytic effect in rodents ${ }^{36,61-63}$. These comparisons must be taken carefully since most of the previous studies have not investigated the long-term effects of gut microbiota depletion. Moreover, a study has found that juvenile rodents react differently to treatment with Lactobacillus, evoking an anxiogenic-phenotype following by changes in the gene expression and brain function ${ }^{64}$, suggesting that the physiological mediators peaking during the adolescent period may be playing a role on the involvement of the Lactobacillus on the gutbrain communication.

It is interesting to note that members of the family Lachnospiraceae (in special NK4A136 group) and Bacteroides genus, which were decreased in $\mathrm{ABX}_{\text {adolescence, }}$ are also associated with anxiety in humans $s^{65,66}$ as well as relevant to the production of SCFAs in rodents ${ }^{67}$. SCFAs are important bacterial metabolites with neuroactive properties $^{67,68}$. Moreover, colonic SCFAs regulate intestinal barrier function ${ }^{69,70}$. Thus, a dysfunction in the intestinal barrier can increase inflammatory response and facilitate that peripheral substances escape into the bloodstream and potentially reach the brain. Further studies are needed to mechanistically unravel the pathways involved.

Interestingly, despite the body of literature showing that the gut microbiota is implicated in social behaviour or cognitive abilities ${ }^{17,20,25,26,50,71-74}$, we did not observe any overt effect of $A B X$ treatment in social behaviour and short-term memory, suggesting that with the parameters we used these phenotypes were not responsive to longlasting effects of gut microbiota depletion. Moreover, it has been shown that deficient synaptic pruning, which is associated with weak synaptic transmission and decreased functional brain connectivity, is correlated with deficits in social interaction and other neuropsychiatric disorders ${ }^{75}$. These failures in the synaptic connectivity are due to the lack of activity of the $C \times 3 c r 1$ gene ${ }^{75,76}$. Cx3cr1 was found upregulated in our study, suggesting a local role on brain inflammatory response which is not directly associated with sociability. In addition, fear conditioning which has previously been shown to be modulated by microbiome manipulations ${ }^{32,36,77}$ was only marginally affected by gut microbiota depletion as only $\mathrm{ABX}_{\text {adolecence }}$ mice showed increased freezing behaviour during fear acquisition, indicating that learning and memory indeed are not overtly influenced by $\mathrm{ABX}$ treatment in this circumstances, despite all the changes observed on the gut microbiota composition. To note, increased levels of Lactobacillus species are described to improve cognition process in rodents, including learning and memory ${ }^{61}$ but not in a clinical study ${ }^{78}$.

Here we focused on the amygdala to understand to which extent changes in gene expression could underly the differences observed in the anxiety-like behaviour. Despite the highest prevalence of overall gene expression changes

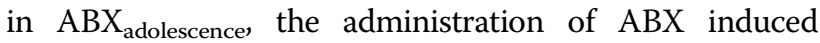
similar disturbance for genes involved in neuroimmunity response in $\mathrm{ABX}_{\text {adolescence }}$ and $\mathrm{ABX}$ adulthood mice. Given the importance of microglia in normal brain function, it is not surprising that these genes have been associated with the ABX treatment. The microglia-related genes $F c g r 2 b$ and $R a c 2$ were upregulated in both groups, while $C \times 3 c r 1$ and C1qa were elevated only in $\mathrm{ABX}_{\text {adolescence }}$ mice, confirming a role for microbiota recolonization observed in $\mathrm{ABX}_{\text {adulthood }}$ (but not in $\mathrm{ABX}_{\text {adolescence }}$ ) with restored microglia features ${ }^{76}$. It has been suggested that elevated levels of the $C x 3 c r 1$ ligand CX3CL1 is associated with proneurogenic response by altering the environment in which new cells are born ${ }^{79}$, suggesting that together with C1qa, Cx3cr1 are playing a role in the prevention of chronic inflammation induced by the gut microbiota depletion. In fact, microglia seems to keep engulfing apoptotic debris even after antibiotic treatment ${ }^{80}$, which together with elevated Tlr4 expression followed by $\mathrm{ABX}_{\text {adolescence }}$ treatment could indicate certain level of neuroinflammation has been occurring without the participation of the cytokines. In 
addition, it is well known that SCFAs directly affect immune response with the intention to maintain homoeostasis by fine-tuning microglial function and production of inflammatory cytokines, further suggesting that the overexpression of the SCFAs transporters Slc5a8 and Slc16a1 in the amygdala of $\mathrm{ABX}_{\text {adolescence }}$ mice could be related with the microbiome-derived factors that are involved in modulate adaptive immune responses in the brain. $C 3$ is a downstream member of the component cascade with an extremely versatile role that it is not restricted to immune responses but also in tissue regeneration and synapse pruning ${ }^{81}$. Interestingly, $C 3$ and $I l 1 \beta$ were elevated only in $\mathrm{ABX}_{\text {adulthood }}$. This could suggest that neuronal loss and consequently cognitive deficits associated with long-term depression caused by $\mathrm{C} 3$ could have been counteracted by the production of $I l 1 \beta$, a cytokine with a neuromodulatory role on neurogenesis ${ }^{82}$.

Tight-junction proteins at the blood-brain barrier are known to be regulated by the gut microbiota, where the decreased expression of these proteins is associated with permeable blood-brain barrier ${ }^{83,84}$. Although gut depletion is necessary to promote tight-junction protein changes in the brain, the direction of these changes is dependent on the brain region and protein tested. Herein, we observed an upregulation of amygdalar Tjp1 and Ocln only in $\mathrm{ABX}_{\text {adolescence }}$ mice, confirming the role of depleted gut microbiota on brain permeability ${ }^{26,84}$. Moreover, this study also showed that the natural recolonization of the gut microbiota is enough to recalibrate the functionality of the information transfer between the gut and brain following a microbiota-targeted insult. Taken together, our findings show that the immunological markers changed in $\mathrm{ABX}_{\text {adolescence }}$ and $\mathrm{ABX}_{\text {adulthood }}$ mice are not a result of a brain leakage through the blood-brain barrier but probably due to local synthesis of volume diffusion into the brain at the circumventricular organs that lack a blood-brain barrier.

When examining the pattern of expression of genes involved in neurotransmission it was clear that they were more strongly affected in $\mathrm{ABX}_{\text {adolescence }}$ than $\mathrm{ABX}_{\text {adulthood }}$ mice. Elevated brain levels of Crh1r has been found after ABX treatment or in GF mice ${ }^{6,20}$ while downregulation of the transcription levels of Crh1r has been found after psychobiotics or SCFAs treatment in stressed animals ${ }^{67,85}$, suggesting that restoration of the normal gut microbiota could explain why we see altered Crh1r expression in $\mathrm{ABX}_{\text {adulthood }}$ mice. In addition, NPY and activation of NPY1R are known to promote stress resilience and have anxiogenic effects ${ }^{86-89}$. NPY locally influences GABAergic activity through NPY1R, and both Npy and Npy1r were found to be upregulated in chronic stress events ${ }^{90}$, in line with the results observed in $\mathrm{ABX}_{\text {adolescence }}$ mice. In fact, evidence suggests that the gut microbiota may contribute to resilience after repeated stress ${ }^{91-93}$. For instance, it has been shown that chronic stress significantly increases gut microbiota diversity in antibiotic-treated mice and can neutralize not only stress-inducing anhedonia phenotypes but also the levels of inflammatory markers, suggesting that the development of susceptibility to stress in mice is subjected to the composition of the gut microbiota ${ }^{93}$. Recently, evidence has also confirmed that the use of commensal bacteria strain to improve psychological functions and cognitive health in stressed adult subjects ${ }^{58}$.

Interestingly, genes related with neuroplasticity, postsynaptic density protein 95 (Psd95) and synaptophysin (Syp) were oppositely affected by $\mathrm{ABX}_{\text {adolescence }}$ and $\mathrm{ABX}_{\text {adulthood, }}$ suggesting a direct role of the gut microbiota composition on the expression of these genes. A recent study has shown that at least in the PFC, ABX treatment protects Psd 95 expression from stress, suggesting that depleted gut microbiota could play a role in the development of susceptibility in mice after stress ${ }^{93}$. However, in this study, stress started after the $\mathrm{ABX}$ treatment, thus investigating the role of stress during the recovery period of the gut microbiota, in agreement with previous literature that found normalized levels of Syp and Psd95 after the restoration of the gut microbiota ${ }^{9}$. Therefore, the upregulated expression of these genes in $\mathrm{ABX}_{\text {adolescence }}$ mice could be due to the long-lasting shift in microbiota composition and structure, whereas the decrease observed in $\mathrm{ABX}_{\text {adulthood }}$ mice might come from an exaggerated downregulation of these genes due to a possible overexpression during the gut microbiota depletion period.

Unexpectedly, $\mathrm{ABX}_{\text {adulthood }}$ treatment seems also to play a role in myelin-related gene. Recent studies have shown upregulation of myelination-related genes in the PFC of animals with disrupted gut microbiota, suggesting a role for the gut microbiota in the formation of myelin ${ }^{37}$. Our study indicates that depletion of the gut microbiota does not exclusively affect myelination during critical developmental periods but can also influence myelinationrelated gene expression in adulthood. Whether these changes in gene expression translates to altered myelination, however, still needs to be investigated.

Overall, this study highlights vulnerability of the gut microbiota during the adolescent period and the importance of the microbiota during the developmental period in shaping its host's microbiota, brain function and behaviour. A limitation of this study is that only males were tested. It is worth pointing out that for the majority of our studies of microbiota to brain interactions the effects are much more robust in males ${ }^{94}$. However, much research is still needed to unravel this interaction, and recent evidence suggests that besides the sex bias for mental disorders, an interaction between the sex, sex hormones and the gut microbiota is highly possible with the gut microbiota ${ }^{94}$. Future studies will determine how sex and sex hormones determine discrete genes changes in the amygdala as well in the gut. Further, 
understanding what pathways of communication between the gut and the brain are responsible for such changes at this time period are also required. Interventions targeting the vagus nerve or the use of specific dietary interventions focused on specific populations of commensal bacteria that are affected by $A B X$ would be of further interest to provide a mechanistic framework of the gut to brain signalling ${ }^{29,95}$. Finally, as adolescence is a time where antibiotics are heavily used clinically ${ }^{28}$, and its use is epidemiologically relevant to the risk of several psychiatric conditions ${ }^{96}$, the results presented here support the need for further studies investigating their impact and their long-term effects on the microbiota and associated risks to the brain function and behaviour.

\section{Acknowledgements}

We thank Dr. Gerard M. Moloney and Mr. Patrick Fitzgerald for technical assistance with animal husbandry, tissue extraction, RNA extraction and GRTPCR. This research was funded by Science Foundation Ireland (SFI/12/RC/2273). APC Microbiome Ireland has conducted research funded by several Pharmaceutical and Food companies. T.G.D. has been an invited speaker at meetings organized by Servier, Lundbeck, Janssen, and AstraZeneca, and has received research funding from Mead Johnson, Cremo, Suntory Wellness, Nutricia and 4D Pharma. J.F.C. has been an invited speaker at meetings organized by Mead Johnson, Yakult, Alkermes, and Janssen, and has received research funding from Mead Johnson, Cremo, Suntory Wellness, Nutricia and 4D Pharma.

\section{Author details}

${ }^{1}$ APC Microbiome Ireland, University College Cork, Cork, Ireland. ${ }^{2}$ Department of Anatomy and Neuroscience, University College Cork, Cork, Ireland. ${ }^{3}$ Teagasc Food Research Centre, Food Biosciences Department, Moorepark, Fermoy, Ireland. ${ }^{4}$ School of Microbiology, University College Cork, Cork, Ireland. ${ }^{5}$ Department of Psychiatry and Neurobehavioural Sciences, University College Cork, Cork, Ireland. 'Present address: University of Edinburgh, Edinburgh, Scotland, UK

\section{Conflict of interest}

The authors declare that they have no conflict of interest.

\section{Publisher's note}

Springer Nature remains neutral with regard to jurisdictional claims in published maps and institutional affiliations.

Supplementary Information accompanies this paper at (https://doi.org/ 10.1038/s41398-020-01073-0).

Received: 21 August 2020 Revised: 15 September 2020 Accepted: 5 October 2020

Published online: 06 November 2020

\section{References}

1. Paus, T., Keshavan, M. \& Giedd, J. N. Why do many psychiatric disorders emerge during adolescence? Nat. Rev. Neurosci. 9, 947-957 (2008).

2. Borre, Y. et al. Microbiota and neurodevelopmental windows: implications for brain disorders. Trends Mol. Med. 20, 509-518 (2014).

3. Fouhy, F., Ross, R. P., Fitzgerald, G. F., Stanton, C. \& Cotter, P. D. Composition of the early intestinal microbiota. Gut Microbes 3, 203-220 (2012).

4. Yurkovetskiy, L. et al. Gender bias in autoimmunity is influenced by microbiota. Immunity 39, 400-412 (2013).

5. Verdú, E. F. et al. Specific probiotic therapy attenuates antibiotic induced visceral hypersensitivity in mice. Gut 55, 182-190 (2006).
6. Neufeld, K-A. M., Luczynski, P., Seira Oriach, C., Dinan, T. G. \& Cryan, J. F. What's bugging your teen? - the microbiota and adolescent mental health. Neurosci. Biobehav. Rev. 70, 300-312 (2016).

7. Codagnone, M. G. et al. Programming bugs: microbiota and the developmental origins of brain health and disease. Biol. Psychiatry 85, 150-163 (2019).

8. Cryan, J. F. et al. The microbiota-gut-brain axis. Physiol. Rev. 99, 1877-2013 (2019).

9. Heijtz, R. D. et al. Normal gut microbiota modulates brain development and behavior. Proc. Natl Acad. Sci. USA 108, 3047-3052 (2011).

10. Lavelle, A. \& Hill, C. Gut microbiome in health and disease: emerging diagnostic opportunities. Gastroenterol. Clin. North Am. 48, 221-235 (2019).

11. Pusceddu, M. M., Murray, K. \& Gareau, M. G. Targeting the microbiota, from irritable bowel syndrome to mood disorders: focus on probiotics and prebiotics. Curr. Pathobiol. Rep. 6, 1-13 (2018).

12. Walsh, C. J., Guinane, C. M., OToole, P. W. \& Cotter, P. D. Beneficial modulation of the gut microbiota. FEBS Lett. 588, 4120-4130 (2014).

13. Cowan, C. S. M., Stylianakis, A. A. \& Richardson, R. Early-life stress, microbiota, and brain development: Probiotics reverse the effects of maternal separation on neural circuits underpinning fear expression and extinction in infant rats. Dev. Cogn. Neurosci. 37, 100627 (2019).

14. Buffington, S. A. et al. Microbial reconstitution reverses maternal diet-induced social and synaptic deficits in offspring. Cell 165, 1762-1775 (2016).

15. Cryan, J. F. \& Dinan, T. G. Mind-altering microorganisms: the impact of the gut microbiota on brain and behaviour. Nat. Rev. Neurosci. 13, 701-712 (2012).

16. Leclercq, S. et al. Low-dose penicillin in early life induces long-term changes in murine gut microbiota, brain cytokines and behavior. Nat. Commun. 8, 15062 (2017).

17. Sgritta, M. et al. Mechanisms underlying microbial-mediated changes in social behavior in mouse models of autism spectrum disorder. Neuron 101, 246-259 (2019).

18. Cox, L. M. et al. Altering the intestinal microbiota during a critical developmental window has lasting metabolic consequences. Cell 158, 705-721 (2014).

19. Ruiz, V. E. et al. A single early-in-life macrolide course has lasting effects on murine microbial network topology and immunity. Nat. Commun. 8, 518 (2017).

20. Hoban, A. E. et al. Behavioural and neurochemical consequences of chronic gut microbiota depletion during adulthood in the rat. Neuroscience 339, 463-477 (2016).

21. Korpela, K. et al. Intestinal microbiome is related to lifetime antibiotic use in Finnish pre-school children. Nat. Commun. 7, 10410 (2016).

22. Ferrer, M., Mendez-Garcia, C., Rojo, D., Barbas, C. \& Moya, A. Antibiotic use and microbiome function. Biochem. Pharm. 134, 114-126 (2017).

23. Luczynski, P. et al. Adult microbiota-deficient mice have distinct dendritic morphological changes: differential effects in the amygdala and hippocampus. Eur. J. Neurosci. 44, 2654-2666 (2016).

24. Lundberg, R., Toft, M. F. M., August, B., Hansen, A. K. \& Hansen, C. H. F. Antibiotic-treated versus germ-free rodents for microbiota transplantation studies. Gut Microbes 7, 68-74 (2016).

25. Desbonnet, L. et al. Gut microbiota depletion from early adolescence in mice: implications for brain and behaviour. Brain Behav. Immun. 48, 165-173 (2015).

26. Fröhlich, E. E. et al. Cognitive impairment by antibiotic-induced gut dysbiosis: analysis of gut microbiota-brain communication. Brain Behav. Immun. 56, 140-155 (2016).

27. O'Mahony, S. M. et al. Disturbance of the gut microbiota in early-life selectively affects visceral pain in adulthood without impacting cognitive or anxietyrelated behaviors in male rats. Neuroscience 277, 885-901 (2014).

28. Balato, A. et al. Human microbiome: composition and role in inflammatory skin diseases. Arch. Immunol. Ther. Exp. 67, 1-18 (2019).

29. Fülling, C. et al. Adolescent dietary manipulations differentially affect gut microbiota composition and amygdala neuroimmune gene expression in male mice in adulthood. Brain Behav. Immun. 87, 666-678 (2020).

30. Dutta, S. \& Sengupta, P. Men and mice: Relating their ages. Life Sci. 152 244-248 (2016)

31. Golubeva, A. V. et al. Microbiota-related changes in bile acid \& tryptophan metabolism are associated with gastrointestinal dysfunction in a mouse model of autism. EBioMedicine 24, 166-178 (2017).

32. Hoban, A. E. et al. The microbiome regulates amygdala-dependent fear recall. Mol. Psychiatry 23, 1-11 (2017).

33. Lach, G., Schellekens, H., Dinan, T. G. \& Cryan, J. F. Anxiety, depression, and the microbiome: a role for gut peptides. Neurotherapeutics 15, 36-59 (2018). 
34. Verma, D. et al. NPY Y2 receptors in the central amygdala reduce cued but not contextual fear. Neuropharmacology 99, 665-674 (2015).

35. Paxinos, G. \& Franklin, K. B. J. The Mouse Brain in Stereotaxic Coordinates 3rd edn (Academic Press, 2008)

36. Bravo, J. A. et al. Ingestion of Lactobacillus strain regulates emotional behavior and central GABA receptor expression in a mouse via the vagus nerve. Proc. Natl Acad. Sci. USA 108, 16050-16055 (2011).

37. Hoban, A. E. et al. Regulation of prefrontal cortex myelination by the microbiota. Transl. Psychiatry 6, e774 (2016).

38. Robinson, $\mathrm{O}$. J. et al. Anxiety-potentiated amygdala-medial frontal coupling and attentional control. Transl. Psychiatry 6, e833-e833. (2016).

39. Stilling, R. M. et al. Social interaction-induced activation of RNA splicing in the amygdala of microbiome-deficient mice. Elife 7, 1-21 (2018).

40. Stilling, R. M. et al. Microbes \& neurodevelopment-absence of microbiota during early life increases activity-related transcriptional pathways in the amygdala. Brain Behav. Immun. 50, 209-220 (2015).

41. Cowan, C. S. M. et al. Gutsy moves: the amygdala as a critical node in microbiota to brain signaling. BioEssays 40, 1-12 (2018).

42. Callahan, B. J. et al. DADA2: high-resolution sample inference from Illumina amplicon data. Nat. Methods 13, 581-583 (2016).

43. Hsieh, T. C., Ma, K. H. \& Chao, A. iNEXT: an R package for rarefaction and extrapolation of species diversity (Hill numbers). Methods. Ecol. Evol. 7, 1451-1456 (2016).

44. Fernandes, A. D., Macklaim, J. M., Linn, T. G., Reid, G. \& Gloor, G. B. ANOVA-like differential expression (ALDEX) analysis for mixed population RNA-Seq. PLoS ONE 8, e67019 (2013)

45. Arentsen, T., Raith, H., Qian, Y., Forssberg, H. \& Heijtz, R. D. Host microbiota modulates development of social preference in mice. Micro. Ecol. Heal Dis. 26, 29719 (2015).

46. Lu, J. et al. Microbiota influence the development of the brain and behaviors in C57BL/6J mice. PLOS ONE 13, e0201829 (2018).

47. Markle, J. G. M. et al. Sex differences in the gut microbiome drive hormonedependent regulation of autoimmunity. Science 339, 1084-1088 (2013).

48. Cowan, C. S. M. \& Richardson, R. Early-life stress leads to sex-dependent changes in pubertal timing in rats that are reversed by a probiotic formulation. Dev. Psychobiol. 61, 679-687 (2019).

49. Hopkins, M. J., Sharp, R. \& Macfarlane, G. T. Variation in human intestinal microbiota with age. Dig. Liver Dis. 34, S12-S18 (2002).

50. Ceylani, T., Jakubowska-Dogru, E., Gurbanov, R., Teker, H. T. \& Gozen, A. G. The effects of repeated antibiotic administration to juvenile BALB/C mice on the microbiota status and animal behavior at the adult age. Heliyon 4, e00644 (2018).

51. Neufeld, K.-A., Luczynski, P., Dinan, T. G. \& Cryan, J. F. Reframing the teenage wasteland: adolescent microbiota-gut-brain axis. Can. J. Psychiatry 61, 214-221 (2016).

52. Mariat, D. et al. The firmicutes/bacteroidetes ratio of the human microbiota changes with age. BMC Microbiol. 9, 123 (2009).

53. Cho, I. et al. Antibiotics in early life alter the murine colonic microbiome and adiposity. Nature 488, 621-626 (2012).

54. Zaura, E. et al. Same exposure but two radically different responses to antibiotics: resilience of the salivary microbiome versus long-term microbial shifts in feces. MBio 6, e01693-15 (2015).

55. Bercik, P. et al. The intestinal microbiota affect central levels of brain-derived neurotropic factor and behavior in mice. Gastroenterology 141, 599-609 (2011).

56. Bercik, P. et al. The anxiolytic effect of Bifidobacterium longum NCC3001 involves vagal pathways for gut-brain communication. Neurogastroenterol. Motil. 23, 1132-1139 (2011).

57. Jang, H. M. M., Jang, S.-E. E., Han, M. J. J. \& Kim, D.-H. H. Anxiolytic-like effect of Bifidobacterium adolescentis IM38 in mice with or without immobilisation stress. Benef. Microbes 9, 1-10 (2017).

58. Chong, H. X. et al. Lactobacillus plantarum DR7 alleviates stress and anxiety in adults: a randomised, double-blind, placebo-controlled study. Benef. Microbes 10, 355-373 (2019).

59. Klarer, M. et al. Gut vagal afferents differentially modulate innate anxiety and learned fear. J. Neurosci. 34, 7067-7076 (2014).

60. Smith, J. A. et al. Hydration and beyond: neuropeptides as mediators of hydromineral balance, anxiety and stress-responsiveness. Front. Syst. Neurosci. 9, 46 (2015)
61. Liang, S. et al. Administration of Lactobacillus helveticus NS8 improves behavioral, cognitive, and biochemical aberrations caused by chronic restraint stress. Neuroscience 310, 561-577 (2015).

62. Wang, T. et al. Lactobacillus fermentum NS9 restores the antibiotic induced physiological and psychological abnormalities in rats. Benef. Microbes $\mathbf{6}$, 707-717 (2015)

63. Takada, M. et al. Probiotic Lactobacillus casei strain Shirota relieves stressassociated symptoms by modulating the gut-brain interaction in human and animal models. Neurogastroenterol. Motil. 28, 1027-1036 (2016).

64. Barrera-Bugueño, C. et al. Anxiogenic effects of a Lactobacillus, inulin and the synbiotic on healthy juvenile rats. Neuroscience 359, 18-29 (2017).

65. Jiang, Hyin et al. Altered gut microbiota profile in patients with generalized anxiety disorder. J. Psychiatr. Res. 104, 130-136 (2018).

66. Tillisch, K. et al. Brain structure and response to emotional stimuli as related to gut microbial profiles in healthy women. Psychosom. Med. 79, 905-913 (2017).

67. van de Wouw, M. et al. Short-chain fatty acids: microbial metabolites that alleviate stress-induced brain-gut axis alterations. J. Physiol. 596, 4923-4944 (2018).

68. Morris, G. et al. The role of the microbial metabolites including tryptophan catabolites and short chain fatty acids in the pathophysiology of immuneinflammatory and neuroimmune disease. Mol. Neurobiol. 54, 4432-4451 (2017).

69. Kelly, J. R. et al. Breaking down the barriers: the gut microbiome, intestinal permeability and stress-related psychiatric disorders. Front Cell Neurosci. 9, 392 (2015).

70. Ait-Belgnaoui, A. et al. Prevention of gut leakiness by a probiotic treatment leads to attenuated HPA response to an acute psychological stress in rats. Psychoneuroendocrinology 37, 1885-1895 (2012).

71. Desbonnet, L., Clarke, G., Shanahan, F., Dinan, T. G. \& Cryan, J. F. Microbiota is essential for social development in the mouse. Mol. Psychiatry 19, 146-148 (2014).

72. Gareau, M. G. et al. Bacterial infection causes stress-induced memory dysfunction in mice. Gut 60, 307-317 (2011).

73. Guida, F. et al. Antibiotic-induced microbiota perturbation causes gut endocannabinoidome changes, hippocampal neuroglial reorganization and depression in mice. Brain Behav. Immun. 67, 230-245 (2018).

74. Haak, B. W. et al. Long-term impact of oral vancomycin, ciprofloxacin and metronidazole on the gut microbiota in healthy humans. J. Antimicrob. Chemother. 74, 782-786 (2019).

75. Zhan, Y. et al. Deficient neuron-microglia signaling results in impaired functional brain connectivity and social behavior. Nat. Neurosci. 17, 400-406 (2014).

76. Erny, D. et al. Host microbiota constantly control maturation and function of microglia in the CNS. Nat. Neurosci. 18, 965-977 (2015).

77. Savignac, H. M., Tramullas, M., Kiely, B., Dinan, T. G. \& Cryan, J. F. Bifidobacteria modulate cognitive processes in an anxious mouse strain. Behav. Brain Res. 287, 59-72 (2015).

78. Kelly, J. R. et al. Lost in translation? The potential psychobiotic Lactobacillus rhamnosus (JB-1) fails to modulate stress or cognitive performance in healthy male subjects. Brain Behav. Immun. 61, 50-59 (2017).

79. Vukovic, J., Colditz, M. J., Blackmore, D. G., Ruitenberg, M. J. \& Bartlett, P. F. Microglia modulate hippocampal neural precursor activity in response to exercise and aging. J. Neurosci. 32, 6435-6443 (2012).

80. Ueno, M. et al. Layer $\vee$ cortical neurons require microglial support for survival during postnatal development. Nat. Neurosci. 16, 543-551 (2013).

81. Zhang, J. et al. Microglial CR3 activation triggers long-term synaptic depression in the hippocampus via NADPH oxidase. Neuron 82, 195-207 (2014).

82. Koo, J. W. \& Duman, R. S. Evidence for IL-1 receptor blockade as a therapeutic strategy for the treatment of depression. Curr. Opin. Investig. Drugs 10, 664-671 (2009).

83. Tietz, S. \& Engelhardt, B. Brain barriers: crosstalk between complex tight junctions and adherens junctions. J. Cell Biol. 209, 493-506 (2015).

84. Braniste, $\mathrm{V}$. et al. The gut microbiota influences blood-brain barrier permeability in mice. Sci. Transl. Med. 6, 263 ra158 (2014).

85. Abildgaard, A., Elfving, B., Hokland, M., Wegener, G. \& Lund, S. Probiotic treatment reduces depressive-like behaviour in rats independently of diet. Psychoneuroendocrinology 79, 40-48 (2017).

86. Verma, D. et al. Hunger promotes fear extinction by activation of an amygdala microcircuit. Neuropsychopharmacology 41, 431-439 (2016). 
87. Tasan, R. O. et al. The role of Neuropeptide $Y$ in fear conditioning and extinction. Neuropeptides 55, 111-126 (2016).

88. Lach, G. et al. Short-term enriched environment exposure facilitates fear extinction in adult rats: the NPY-Y1 receptor modulation. Neuropeptides $\mathbf{5 5}$ 73-78 (2016).

89. Lach, G. \& de Lima, T. C. M. Role of NPY Y1 receptor on acquisition, consolidation and extinction on contextual fear conditioning: Dissociation between anxiety, locomotion and non-emotional memory behavior. Neurobiol. Learn Mem. 103, 26-33 (2013).

90. Thorsell, A., Carlsson, K., Ekman, R. \& Heilig, M. Behavioral and endocrine adaptation, and upregulation of NPY expression in rat amygdala following repeated restraint stress. Neuroreport 10, 3003-3007 (1999).

91. Yang, C. et al. R-ketamine: a rapid-onset and sustained antidepressant without psychotomimetic side effects. Transl. Psychiatry 5, e632 (2015).
92. Yang, $C$. et al. Possible role of the gut microbiota-brain axis in the antidepressant effects of (R)-ketamine in a social defeat stress model. Transl. Psychiatry 7, 1294 (2017)

93. Wang, S. et al. Antibiotic-induced microbiome depletion is associated with resilience in mice after chronic social defeat stress. J. Affect Disord. 260, 448-457 (2020)

94. Jaggar, M., Rea, K., Spichak, S., Dinan, T. G. \& Cryan, J. F. You've got male: sex and the microbiota-gut-brain axis across the lifespan. Front. Neuroendocrinol. 56, 100815 (2020)

95. Fülling, C., Dinan, T. G. \& Cryan, J. F. Gut microbe to brain signaling: what happens in vagus.... Neuron 101, 998-1002 (2019).

96. Lurie, I., Yang, Y.-X., Haynes, K., Mamtani, R. \& Boursi, B. Antibiotic exposure and the risk for depression, anxiety, or psychosis. J. Clin. Psychiatry 76, 1522-1528 (2015). 